\title{
ORIENTACIÓN DEL CURRÍCULO EN LOS ESTUDIOS DE PREGRADO DE LA UNIVERSIDAD DE CHILE
}

Sergio Gómez D.* 


\section{RESUMEN}

El presente artículo describe los lineamientos centrales de la reforma de los Estudios de Pregrado de la Universidad de Chile, centrados en el fortalecimiento de la calidad y la equidad de la educación universitaria, con el propósito de responder a las demandas que plantea el nuevo contexto en que se inserta nuestro país. Estas demandas se expresan en el requerimiento de un profesional universitario con un nuevo perfil constituido por competencias que le permitan un desempeño futuro en un ambiente de cambio permanente, alta competitividad y exigencias de una educación continua. Se aborda la nueva estructura curricular, sus principios y marco normativo, junto con los desafíos pendientes que caracterizan a un proceso en marcha.

\section{ABSTRACT}

The paper describes the main guidelines of the reform of the Undergraduate Studies program at the Universidad de Chile. This has centered on strengthening the quality and equity of university education in order to respond to the demands required by the new context in which our country is operating. These demands call for university professionals who have a profile consisting of competencies that will allow them to perform in future in a constantly changing setting which will be marked by high competitiveness and demands for continuing education. The new curricular structure is taken into account, as are its principles and regulatory standards, together with the outstanding challenges that characterize an ongoing process. 


\section{ORIENTACIÓN DEL CURRÍCULO EN LOS ESTUDIOS DE PREGRADO DE LA UNIVERSIDAD DE CHILE}

\section{REFORMA DE LOS ESTUDIOS DE PREGRADO}

\section{A) Fundamentación}

Es conocida la importancia que han cobrado el fenómeno de la globalización y sus efectos en relación con los desafíos que representan para nuestra sociedad. Por una parte, la multiplicación exponencial de los medios infocomunicacionales ha permitido la circulación de un acervo de información que crece día a día; por otra, los procesos de modernización técnica vienen asociados a un conjunto de fenómenos que deben ser atendidos, a fin de dar cuenta real de la situación a partir de la cual nos insertamos en la mundialización. Uno de los requerimientos es la capacidad de iniciativa que tenemos al interior de esos procesos y otro, la posibilidad de aprovechar las instancias de participación en la sociedad global para obtener un desarrollo que se traduzca en provecho social.

Entre los problemas principales que determinan nuestra desventaja para llevar a cabo adecuadamente esa enorme tarea se encuentra una serie de deficiencias en el capital humano nacional, en términos de la calidad de la educación y la oferta de educación continua. Esto se observa, por ejemplo, en falencias como el manejo de información escrita o la debilidad existente en el área matemática y científica. Todo ello se torna altamente relevante en un contexto cuyo eje central es la productivización de la información, y nos impone el desafío de innovar para lograr un equilibrio entre sistema educativo y demandas contextuales. Los múltiples procesos mundializadores que conforman la sociedad de la información nos obligan a convertir nuestra realidad cultural en una sociedad del aprendizaje, tarea que recién comenzamos a abordar y que nos permitirá llegar a hablar con propiedad de una "sociedad del conocimiento". 
La relación entre las tareas y el rendimiento de la Universidad y las necesidades y expectativas de la sociedad en la que está inserta, es un principio que concierne de la manera más profunda a la vocación histórica de la Universidad de Chile. Nuestra docencia debe establecer las bases de una formación que prepare a las personas para la reflexión y la acción creadora y crítica en su medio, exigencia que repercute en las decisiones sobre el concepto articulador de la enseñanza.

Hasta hoy, la enseñanza impartida por la Universidad de Chile ha sido uno de los pilares de su prestigio académico, tanto por la calidad de los investigadores y profesionales graduados como por la docencia que actualmente brinda a sus estudiantes. En el transcurso de su historia, las diversas facultades de la Universidad de Chile han tenido una gravitación señera en el ejercicio de los respectivos dominios disciplinales y profesionales en el país, lo que ha estado en la esencia misma de la Universidad, representada en su proyección a la vida nacional. El sentido de la reforma debe ir encaminado a fortalecer esta presencia y liderazgo de nuestra Universidad.

Desde una perspectiva histórica, podríamos decir que la reforma afecta, desde el punto de vista docente, al modelo mismo de Universidad: frente al viejo paradigma de la universidad enciclopédica y frente al paradigma más reciente de la universidad profesionalizante, se trata de configurar otro que, sin descuidar la solvencia cognoscitiva y la competencia profesional, ponga énfasis preferente en la formación de sujetos reflexivos, responsables, críticos y versátiles. Si la universidad enciclopédica se ha vuelto disfuncional debido a la aceleración del desarrollo y a la consiguiente obsolescencia de los conocimientos, la universidad profesional, al margen de un sinnúmero de efectos colaterales, tiene al menos dos debilidades básicas que el contexto actual hace cada vez más evidentes: tiende a la rigidez del proceso educativo y a la restricción de las opciones vocacionales y laborales de sus destinatarios.

Para contrarrestar lo anterior, la reforma proyectada está orientada, entonces, a diseñar e implementar la enseñanza de pregrado como un proceso continuo, articulado, dúctil y coherente, que cautela la equidad y garantiza una enseñanza de alta calidad, a fin de preparar al estudiante para un desempeño superior en el dominio disciplinal o 
en el ámbito profesional en el cual se ha formado. Consecuentemente, los principios que la sustentan son:

- Flexibilizar los planes y programas de estudio, a fin de permitir al estudiante una familiarización adecuada con diversos campos y áreas del saber, una eficaz exploración vocacional y una efectiva posibilidad de concretar las opciones que surjan de ésta;

- Disponer de una organización transversal de los estudios, que regule la movilidad dentro de un área de conocimiento y entre áreas, facilitando las homologaciones y transferencias, y disminuyendo la extensión innecesaria de los estudios y los índices de deserción;

- Procurar que el estudiante tenga un rol protagónico en el proceso formativo, atendiendo a sus características intelectuales y afectivas, así como al fomento de un sentido de responsabilidad social y pertenencia institucional, e incentivando el espíritu crítico e investigativo;

- Favorecer la exploración, experimentación y aplicación de metodologías y tecnologías docentes, que renueven el proceso de transmisión y adquisición de conocimientos y destrezas e incorporen actividades orientadas al desarrollo integral y al sentido de convivencia universitaria;

- Propender a criterios y sistemas de autoevaluación periódica de la formación de pregrado;

- Recuperar el valor central que la docencia de pregrado debe tener como función académica preferente;

- Tender a una mayor pertinencia de la formación universitaria en relación con las necesidades y expectativas de la comunidad nacional.

\section{B) Contextualización normativa.}

Con vistas a la paulatina aplicación de dichos principios y orientaciones, el Departamento de Pregrado de la Vicerrectoría de Asuntos Académicos ha planteado su concreción y oficialización, en una primera instancia, mediante la aprobación del nuevo "Reglamento de los Estudios Universitarios de Pregrado", aprobado a comienzos del año 2001. Este cuerpo normativo establece una estructuración en la que se distinguen los diversos tipos y niveles de la formación de pregrado, estableciendo tres áreas interdependientes: formación 
general, formación básica y formación especializada. Estas áreas facilitarán una nueva comprensión de la organización curricular que responda a las demandas de actualización, flexibilidad y transversalidad para la formación de graduados y profesionales, capacitados para liderar, tanto en el campo laboral como en las diversas instancias de postgrado y postítulo, en un contexto signado por el imperativo de la educación continua.

Aunque en esta misma publicación se tratan separadamente las dos primeras áreas de formación mencionadas, viene al caso señalar aquí lo medular que las distingue:

1) El área de formación general contribuye al desarrollo pluralista, equilibrado e integral del estudiante, lo que le permite ser un agente activo en el desarrollo social, desde la perspectiva del desempeño ético de su profesión u ocupación. Más específicamente, la formación general está constituida por un conjunto de contenidos que conciernen a la diversidad de las relaciones entre el ser humano y la realidad, en la medida que éstas requieren una orientación reflexiva y cognoscitiva;

2) El área de formación básica proporciona a los estudiantes los conocimientos, habilidades y destrezas indispensables para la correcta comprensión de un sector del conocimiento y la tecnología. Está integrada por aquellos cuerpos de conocimientos, enfoques metodológicos y estrategias de indagación que, dotadas de estabilidad epistemológica, definen la identidad de una disciplina, y por aquellas otras que informan de manera comparable a una diversidad de disciplinas afines al interior de un área del saber;

3) El área de la formación especializada procura los conocimientos, habilidades, destrezas y actitudes vinculadas directamente con la preparación profesional o académica especializada, indispensable para actuar en la solución de problemas propios de un determinado sector del conocimiento y de las técnicas involucradas en el quehacer profesional. En consecuencia, cabe asociar la formación especializada con aquel cuerpo de conocimientos, metodologías, destrezas y tecnologías fundamentales que son pertinentes al estado actual de desarrollo de una disciplina o profesión, y cuya apropiación permite al futuro egresado adquirir las condiciones epistemológicas y prácticas que aseguren el buen desempeño y/o 
el avance ulterior en una y otra, según el principio de la educación continua.

El cumplimiento de una tarea de esta magnitud implica una fina combinación de prudencia y de audacia, un repertorio de principios, criterios y conceptos rigurosamente fundamentados, un conocimiento preciso de las diversas realidades particulares y la imprescindible visión y decisión estratégica de largo alcance de las autoridades de la Universidad.

Complemento necesario de la nueva organización curricular es la modificación del Reglamento General de Estudiantes Universitarios de Pregrado, en el sentido de normar el reconocimiento de asignaturas y la transferencia entre carreras o programas. En relación con el primer tema, se distingue entre convalidación, homologación y validación; se definen estos mecanismos y se determinan exigencias mínimas, las que pueden ser complementadas por las facultades con mayores exigencias y complejidad. El otro tema -transferencias entre carreras y programas académicos- permite diferenciar entre cambios al interior de la Universidad o desde otra, determinando las condiciones y exigencias curriculares.

Estas definiciones normativas facilitan la transversalidad de los estudios, orientada por mecanismos de reconocimiento curricular consensuados y posibilitan, asimismo, las transferencias entre carreras y programas académicos de una misma área del conocimiento o entre áreas diferentes, lo que, a no dudarlo, elevará nuestras tasas de retención mediante opciones curriculares más amplias.

\section{AVANCES DE LA REFORMA DE PREGRADO}

La reforma de los Estudios de Pregrado de la Universidad de Chile aspira a responder a la indeterminación y al desarrollo vocacional de los estudiantes, a sus diferentes necesidades académicas, tales como la movilidad transversal, y a las necesarias homologaciones o a la posibilidad de opciones de formación complementaria, constituyendo núcleos que podrían obtener un reconocimiento oficial expresado en alguna forma de certificación o grado académico intermedio. Se trata, 
en fin, de abrir nuevas perspectivas en la formación de pregrado y de propiciar otras y nuevas opciones vocacionales.

Un avance hacia la materialización de estos esfuerzos ha sido, con la permanente colaboración del Departamento de Pregrado, la concreción de cambios curriculares en varias carreras de nuestra corporación. A título de ejemplo, hemos tomado dos que se explicitan a continuación:

a) Modificación del Reglamento y Plan de Estudios de la carrera de Administración Pública, caracterizada por la participación de toda la comunidad y porque no sólo rescata sus visiones, sino también una diversidad importante de estudios anteriores.

La revisión de los contenidos, por una parte, y la estructuración de la malla curricular en función del concepto de competencias, por otra, son los ejes centrales de esta flexibilización, la que tiende a fortalecer tanto el sentido académico como profesional de la formación universitaria del administrador público.

En cuanto al primer eje, se perfilaron los contenidos de las actividades curriculares en búsqueda de los elementos epistemológicamente fundamentales, mediante criterios de pertinencia y relevancia, evitando el exceso de formación especializada para permitir la potenciación de la formación básica y la introducción de la línea de formación general. En cuanto al segundo eje, se buscó la mayor coherencia con el perfil de egreso, potenciando la multidisciplinariedad propia de la carrera, a la vez que acotando su objeto de estudio.

Se logró, asimismo, una mayor flexibilidad de los estudios gracias a la reducción de los requisitos previos de las asignaturas a los estrictamente necesarios, a la semestralización de aquéllas y a la desaparición de los exámenes de fin de semestre, mediante el fortalecimiento del proceso evaluativo durante el desarrollo de la docencia.

b) Otro ejemplo destacado en materia de adecuación curricular a las nuevas orientaciones de los estudios de pregrado, lo constituye el radical cambio en la carrera de Derecho, que a la fecha se regía por 
el plan de estudios más antiguo de la Universidad. Contrariamente a lo que pudiera pensarse, este cambio no obedeció sólo a la necesidad de actualización que ha impuesto la Reforma Judicial, sino más bien a un sostenido proceso de reflexión llevado a cabo por los académicos de esta Facultad, con el afán de responder adecuadamente a los desafíos que impone la formación jurídica en el nuevo contexto de la educación superior.

Entre los cambios más notables se encuentra la total semestralización de las actividades curriculares, cuya carga de trabajo, al igual que en otras carreras de pregrado que se encuentran modificando sus mallas curriculares, comienza a regularse en mínimos y máximos de créditos inscritos, es decir, considerando horas docentes y trabajo personal del estudiante en cada semestre. A esto se agrega una mayor autonomía del estudiante en la inscripción de la carga académica, gracias a la reducción de los requisitos de asignatura y a la posibilidad de profundizar en líneas temáticas de su interés; de esta forma se supera la perspectiva enciclopedista que ha caracterizado a la formación jurídica en nuestro país, dándole una dinámica curricular notable.

La estructura del plan de estudios, de esta suerte, permite un amplio grado de decisión curricular al estudiante. La heterogeneidad que pudieran producir estos cauces se equilibra mediante una oferta permanente y una oferta variable de cursos electivos, que aseguran la estabilidad de su calidad académica y la equidad en su formación.

\section{UNA VISIÓN PROSPECTIVA}

El propósito que se persigue con la rearticulación de la enseñanza de pregrado es, junto con asegurar la coherencia y flexibilidad de los estudios, garantizar los más altos estándares de calidad en la docencia que la Universidad imparte. Se estimula así el concurso de sus académicos más relevantes, desde los primeros niveles, en el diseño y ejecución de las asignaturas fundamentales y mediante mecanismos de reconocimiento de la función docente: para ello se establecen vínculos sustantivos entre el compromiso disciplinal que se verifica en la investigación, la creación, la solvencia y el liderazgo profesional 
con el ejercicio docente, entendiendo que aquéllos representan el principal fundamento de una enseñanza capaz de satisfacer las más elevadas exigencias de jerarquía. Entendemos que todos los programas docentes de la Universidad, también aquellos que gozan de notoria firmeza y probada competencia, deberían verse potenciados con esta propuesta.

Con base en los antecedentes recogidos y en las diversas opiniones de la comunidad universitaria se postula llevar a cabo una organización general de los estudios de pregrado, que contemple el ajuste de las mallas curriculares, una distribución porcentual de los distintos tipos de formación, una semestralización de todas las asignaturas y su expresión en horas docentes y en créditos, señalando formas de ingreso, grados intermedios, grados terminales y títulos. Se estima que este proceso debería estar finiquitado dentro de 4 ó 5 años, extendiéndose a todos los programas de la Universidad, los que deberían adecuarse en concordancia con los principios, criterios y lineamientos aquí expuestos.

Se estima que la formación general debiera tener un "créditaje" del orden del $10 \%$ del total y la formación básica no menos de un $40 \%$, ajustando ambas a los requerimientos específicos de las disciplinas y profesiones. Se plantea el régimen semestral, ampliamente mayoritario, para lograr la flexibilidad y movilidad deseadas. Respecto del ingreso se ha descartado, por ahora, un posible ingreso común, por la enorme diversidad temática que contempla nuestra Universidad. En el mediano o en el largo plazo podría proyectarse un ingreso por áreas, siempre que la maduración del proceso de reforma suministrase sólidos argumentos para ello. Cabe indicar que este criterio no obsta a la existencia de planes comunes de ingreso, como existe en Ciencias Físicas y Matemáticas o en el Campus Sur, ni la creación de los mismos en las áreas en que se juzgue adecuado.

Una iniciativa interesante es la de crear dos clases de grado de Bachiller: uno por áreas disciplinales y el otro, que corresponde al actual Programa de Bachillerato, como un Bachillerato General. El primero se obtendría cuando el estudiante haya cursado una cantidad, por determinar en cada carrera, de créditos en asignaturas de cada uno de los tres tipos de formación definidos. Este grado sería opcional 
y se podría obtener a partir del segundo año de cada licenciatura, dependiendo del currículo que cada estudiante haya definido como prioritario. Este Bachillerato por área será una opción para estudiantes que no tienen clara su vocación por la carrera a la que han ingresado, y les serviría para adquirir una formación general y básica amplia y para facilitar una posible transferencia a otras carreras del área.

En virtud de la oferta de asignaturas electivas y de la definición de líneas de formación, también sería posible obtener menciones de formación complementaria, en función de que se cumpla con los requisitos que se establezcan para cada caso.

Respecto de las licenciaturas, pensamos que, sin perjuicio de las actualmente existentes, debería haber una tendencia a reconvertirlas o a instaurar licenciaturas también de área, una vez cumplidos los "creditajes" que se definan para cada programa. Ello permitiría ofrecer una adecuada alternativa a los estudiantes que aspiran a proseguir una labor académica o de investigación básica.

Finalmente, es preciso ahondar en instancias y medios de actualización y renovación de la docencia en lo que atañe a metodologías, estrategias y recursos tecnológicos. Se hace necesario, en consecuencia, favorecer la investigación, experimentación y aplicación de metodologías y tecnologías docentes, que renueven el proceso de transmisión y adquisición de conocimientos y destrezas, estimulen el autoaprendizaje e incorporen actividades orientadas al desarrollo personal integral y al sentido de convivencia universitaria, como asimismo de responsabilidad social. 